\section{MR Spectroscopy of a Bright Cerebellar Lesion in a Patient with Langerhans Cell Histiocytosis}

We have read with interest the paper by Prosch et al ${ }^{1}$ published in the American Journal of Neuroradiology concerning the long-term MR imaging course of 9 patients diagnosed with Langerhans cell histiocytosis $(\mathrm{LCH})$. We were particularly interested in the evolution of MR abnormalities of cerebellum, pons, and basal ganglia structures. The authors observed no correlation between the progression of such abnormalities and clinical neurologic deterioration, and stated that the signal intensity persistence and progression were compatible with a neurodegenerative process, as reported by previous histopathologic studies. Furthermore, symmetric hyperintensities on T1-weighted images observed in these patients were first reported in other diseases, such a chronic liver failure. Such abnormalities could be found in up to $10 \%$ of patients with $\mathrm{LCH}$. We have had the opportunity to study a patient similar to those presented in their report, a 3-year-old boy who was diagnosed with $\mathrm{LCH}$ because of diabetes insipidus and was treated only with antidiuretic hormone replacement and followed, both clinically and radiologically, over a course of 7 years. In the first MR imaging study, abnormalities encountered in our patient were those typically found in the pituitary-hypothalamic area, in patients with LCH and positive granulomata involvement, consisting of a lack of a hyperintense neurohypophyseal spot on noncontrast T1weighted images and thickening and enhancement of the pituitary stalk and adjacent hypothalamic area. During this period of time, the MR enhancing pituitary lesions resolved with almost complete resolution. However, new progressive different lesions appeared, mainly in the basal ganglia and cerebellum, similar to those described in the report by Prosch et al. ${ }^{1}$ In the last study, we also performed monovoxel (TE, $35 \mathrm{~ms}$ ) and multivoxel (TE, $144 \mathrm{~ms}$ ) ${ }^{1} \mathrm{H}-\mathrm{MR}$ spectroscopy on the cerebellar lesions (point-resolved spectroscopy sequence acquisition; Fig 1). The spectrum showed a marked decrease of the $\mathrm{N}$-acetylaspartate peak and an increase of the choline, as the most important findings. These findings would correlate with the histopathologic results found in other patients referred with similar lesions ${ }^{2}$ and would fit with the histopathologic findings of loss of neurons, demyelination, gliosis, and inflammation. Such a histopathologic-spectroscopic correlation has not been reported previously.

\section{References}

1. Prosch H, Grois N, Wnorowski M, et al. Long-term MR imaging course of neurodegenerative Langerhans cell histiocytosis. AJNR Am J Neuroradiol 2007;28:1022-28

2. Gros N, Prayer D, Prosch H, et al. Neuropathology of CNS disease in Langerhans cell hystiocytosis. Brain 2005;128:829-38

A. Munoz

Pediatric Neuroradiology

R. Simon

Pediatric Neurology

G. Carmen

Pediatric Endocrinology

Hospital Universitario "12 Doce de Octubre"

Universidad Complutense

Madrid, Spain

DOI 10.3174/ajnr.A0911
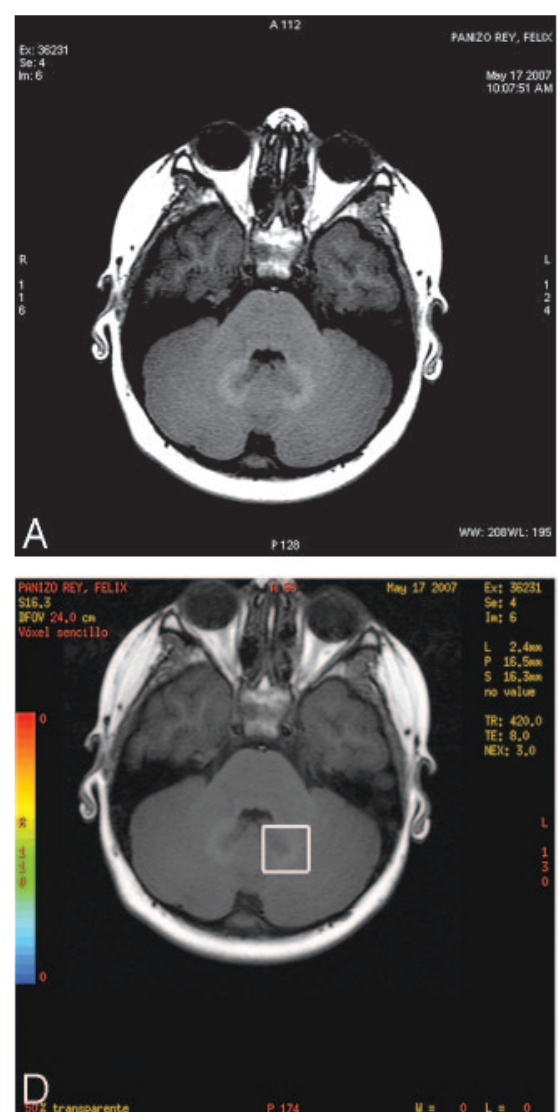


Fig 1. A, Axial noncontrast T1-weighted (500/12, TR/TE) MR image through the cerebellum shows hyperintense abnormal signal intensity lesions on both dentate nuclei of cerebellum. $B-D$, Monovoxel (B) ${ }^{1} \mathrm{H}-\mathrm{MRS}$ spectrum (TE, $35 \mathrm{~ms}$ ) and multivoxel $(C$; TE, $144 \mathrm{~ms}$ ) taken from the left dentate nucleus $(D)$ shows a prominent Cho peak and decrease of the NAA peak. 